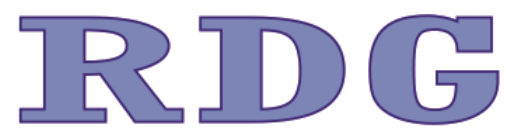

Revista do Departamento de Geografia USP
Revista do Departamento de Geografia

Universidade de São Paulo

www.revistas.usp.br/rdg

ISSN 2236-2878

V.34 (2017)

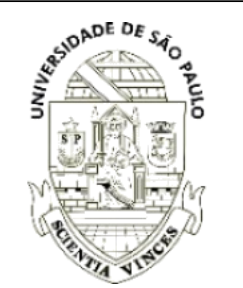

\title{
Uso de Modelo Aditivo Generalizado para \\ Análise Espacial da Suscetibilidade a Movimentos de Massa
}

\section{Generalized Additive Model for Spatial Analysis of Mass Movements Susceptibility}

\author{
Camile Sothe \\ Instituto Nacional de Pesquisas Espaciais \\ camile.sothe@inpe.br \\ Eduardo Celso Gerbi Camargo \\ Instituto Nacional de Pesquisas Espaciais \\ eduardo.camargo@inpe.br \\ Jéssica Gerente \\ Instituto Nacional de Pesquisas Espaciais \\ jessica.gerente@inpe.br \\ Camilo Daleles Rennó \\ Instituto Nacional de Pesquisas Espaciais \\ camilo@dpi.inpe.br \\ Antônio Miguel Vieira Monteiro \\ Instituto Nacional de Pesquisas Espaciais \\ miguel@dpi.inpe.br
}

Resumo: Neste artigo, é analisada a distribuição espacial da suscetibilidade a movimentos de massa da Bacia Hidrográfica do Rio Luís Alves, localizada no estado de Santa Catarina. A modelagem empregada baseia-se em processos pontuais espaciais, na qual se define uma medida de suscetibilidade que varia continuamente sobre a região de estudo e é estimada por meio de métodos de modelos aditivos generalizados (GAM). A suscetibilidade a movimentos de massa, neste contexto, é quantificada por níveis de probabilidades. O procedimento empregado incorpora ao modelo fatores condicionantes de suscetibilidade, de forma simples e de fácil interpretação. O método viabiliza a construção de superfícies de decisão que permitem a geração de mapas com contornos de tolerância baseado em medidas de probabilidade. Tais mapas auxiliam na identificação de áreas de alta/baixa suscetibilidade, uma vez que a hipótese nula de suscetibilidade constante na região de estudo pode ser testada. O resultado da aplicação do modelo mostrou que a variação espacial da suscetibilidade na área de estudo foi significativa a certos fatores condicionantes, apontando um caminho para avanços nos sistemas técnicos de monitoramento e alerta a estas situações, e ampliando as possibilidades para as decisões necessárias que possam minimizar os impactos de processos geomorfológicos danosos, tais como movimentos de massa.

Palavras-Chave: Bacia Hidrográfica do Rio Luís Alves; Mapeamento de Suscetibilidade; Processos Pontuais Espaciais
Abstract: This paper analyzes spatial distribution of mass movements susceptibility from Luís Alves watershed, Santa Catarina State, Brazil. The modeling framework adopted in this research is based on spatial point processes, which defines a susceptibility measure that varies continuously over the study region and is estimated by means of generalized additive modeling methods. In this paper, the mass movements susceptibility is quantified by probability levels. The procedure employed allows susceptibility factors to be incorporated into the model in a simple way and easy interpretation. The procedure also allows the construction of maps with tolerance contours which help identify areas of significantly high/low susceptibility and an overall test for the null hypothesis of constant risk over the region. The application of the model to the data of susceptibility to mass movements, presented results consistent with the geomorphology of the study region, showed that the spatial variation in the susceptibility is significant, and pointing a way to the advance of monitoring and decisions making support systems.

Keywords: Luís Alves watershed; Susceptibility Mapping; Spatial point processes. 


\section{INTRODUÇÃO}

Os movimentos de massa são processos modeladores das encostas, fazendo parte da sua dinâmica natural. Eles podem ser desencadeados por diferentes fatores condicionantes, tais como geologia, características da precipitação, geometria das encostas, natureza dos materiais que compõem as formações superficiais, entre outros (SELBY, 1993). Esses fenômenos são classificados de acordo com uma série de características, variando conforme o tipo e a velocidade de deslocamento do material envolvido. Apesar de serem desencadeados por uma série de fatores naturais, podem ser potencializados pela ação antrópica, principalmente por meio de ações que modifiquem a geometria da encosta, como por exemplo, cortes em terrenos (PINTO et al., 2012).

No Brasil, assim como em muitos países de clima tropical úmido, a ocorrência de eventos relacionados a movimentos de massa está fortemente associada a períodos de precipitação intensa e/ou extrema, causando prejuízos econômicos e, principalmente, perda de vidas humanas. De um modo geral, população, autoridades, instituições de pesquisa e entidades componentes dos arranjos necessários à construção da preparação e prevenção ainda não conseguiram estabelecer diálogos efetivos para transformar conhecimento em ação (GALL et al., 2015).

Os avanços nos sistemas de informação de base territorial e nas tecnologias associadas a coleta, armazenamento, processamento e disseminação de dados espacialmente referenciados, tornou possível o uso de recursos técnicos e métodos mais elaborados para os estudos associados a caracterização de suscetibilidades da paisagem a movimentos de massa. Isto permite, em relação aos sistemas técnicos, a criação de instrumentos mais robustos e adequados, que podem auxiliar pesquisadores e administradores públicos na busca de uma melhor compreensão dos processos associados aos eventos e, assim, pensar em estratégias de adaptação, mitigação ou prevenção. É neste sentido, que o presente trabalho se insere. Aplica uma metodologia de modelagem espacial, baseada em processos pontuais espaciais, para a análise de eventos relacionados a movimentos de massa.

Uma breve revisão na literatura destaca algumas abordagens utilizadas para a investigação e modelagem de eventos associados a movimentos de massa (GUZZETTI et al., 1999; BRENNING, 2005; VORPAHL et al., 2012; BASTOS e PEULVAST, 2016). Entre elas, os modelos estatísticos são apontados como meios eficientes para identificar áreas com alto potencial de deflagração desse fenômeno, permitindo medir as probabilidades associadas a fatores condicionantes à ocorrência de movimentos de massa (BRENNING, 2012). Esses modelos geralmente partem do princípio de que a ocorrência de novos eventos é baseada em condições semelhantes de eventos anteriores.

Um dos modelos estatísticos comumente utilizado é o Modelo Linear Generalizado - GLM (AYALEW e YAMAGISHI, 2005; VAN DEN EECKHAUT et al., 2006). Porém, alguns estudos indicam restrições impostas na aplicação dessa abordagem, como a possível presença de um relacionamento não linear entre as variáveis e a necessidade de lidar com a autocorrelação espacial do fenômeno investigado (GOETZ et al., 2011; BRENNING, 2012; VORPAHL et al., 2012).

Para suprir as restrições impostas pelo GLM, a literatura sugere o uso dos denominados Modelos Aditivos Generalizados - GAM (HASTIE e TIBSHIRANI, 1990). Em síntese, o GAM é uma extensão do GLM, no qual os coeficientes lineares de cada variável explicativa são substituídos por funções de alisamento ajustadas empiricamente (ex: splines), permitindo expressar as relações não lineares entre as variáveis resposta e explicativa. Algumas aplicações do uso do GAM para a modelagem da suscetibilidade a movimentos de massa podem ser encontradas em Brenning (2008), Park e Chi (2008), Goetz et al. (2011), Vorpahl et al. (2012) e Petschko et al. (2014). Neste ponto, vale ressaltar que o uso de modelos estatísticos como o GAM, para estudos da suscetibilidade a movimentos de massa, é incipiente no Brasil. Em razão disso, torna-se atraente a realização de pesquisas que analisem as potencialidades e limitações deste modelo para tal aplicação e área de estudo.

Kelsall e Diggle (1998) propõem uma metodologia de modelagem para análise dos dados baseada na teoria de processos pontuais espaciais. Sob esta estrutura de modelagem, define-se uma medida de risco, que varia continuamente sobre a região de estudo e que pode ser estimada por intermédio de modelos aditivos generalizados. 
Neste artigo, a metodologia estatística proposta por Kelsall e Diggle (1998) foi adaptada e aplicada para investigação e modelagem da suscetibilidade a movimentos de massa na Bacia Hidrográfica do Rio Luís Alves, localizada no estado de Santa Catarina. No contexto deste trabalho, a suscetibilidade foi modelada para os movimentos de massa ocorridos na área de estudo em novembro de 2008 . Na ocasião, fortes precipitações causaram diversos movimentos de massa, dentre eles: deslizamento translacional, deslizamento rotacional e corridas de detritos que, neste trabalho, são abordados sem distinção por tipologia. A metodologia empregada possui a vantagem de permitir incorporar ao modelo fatores condicionantes de movimentos de massa de forma simples. Além disso, possibilita a construção de mapas de suscetibilidade a movimentos de massa com contornos de tolerância, estabelecidos pelas medidas de probabilidades, que auxiliam na delimitação das localidades, distribuídas pela extensão da bacia, com maiores e menores chances de ocorrência para movimentos de massa.

\section{2. ÁREA DE ESTUDO}

A Bacia Hidrográfica do Rio Luís Alves está situada ao nordeste do estado de Santa Catarina, com área aproximada de $592 \mathrm{~km}^{2}$. Engloba o município de Luís Alves e abrange parte dos municípios de Ilhota, Barra Velha, Massaranduba, Navegantes, Balneário Piçarras e São João do Itaperiú (Figura 1). Segundo Köppen, a classificação climática da região é do tipo clima subtropical mesotérmico. $A$ área sofre atuação de frentes frias, ciclones extratropicais, vórtices ciclônicos e sistemas convectivos de mesoescala (CARDOSO et al., 2008), o que contribui para frequentes inundações e movimentos de massa na região.

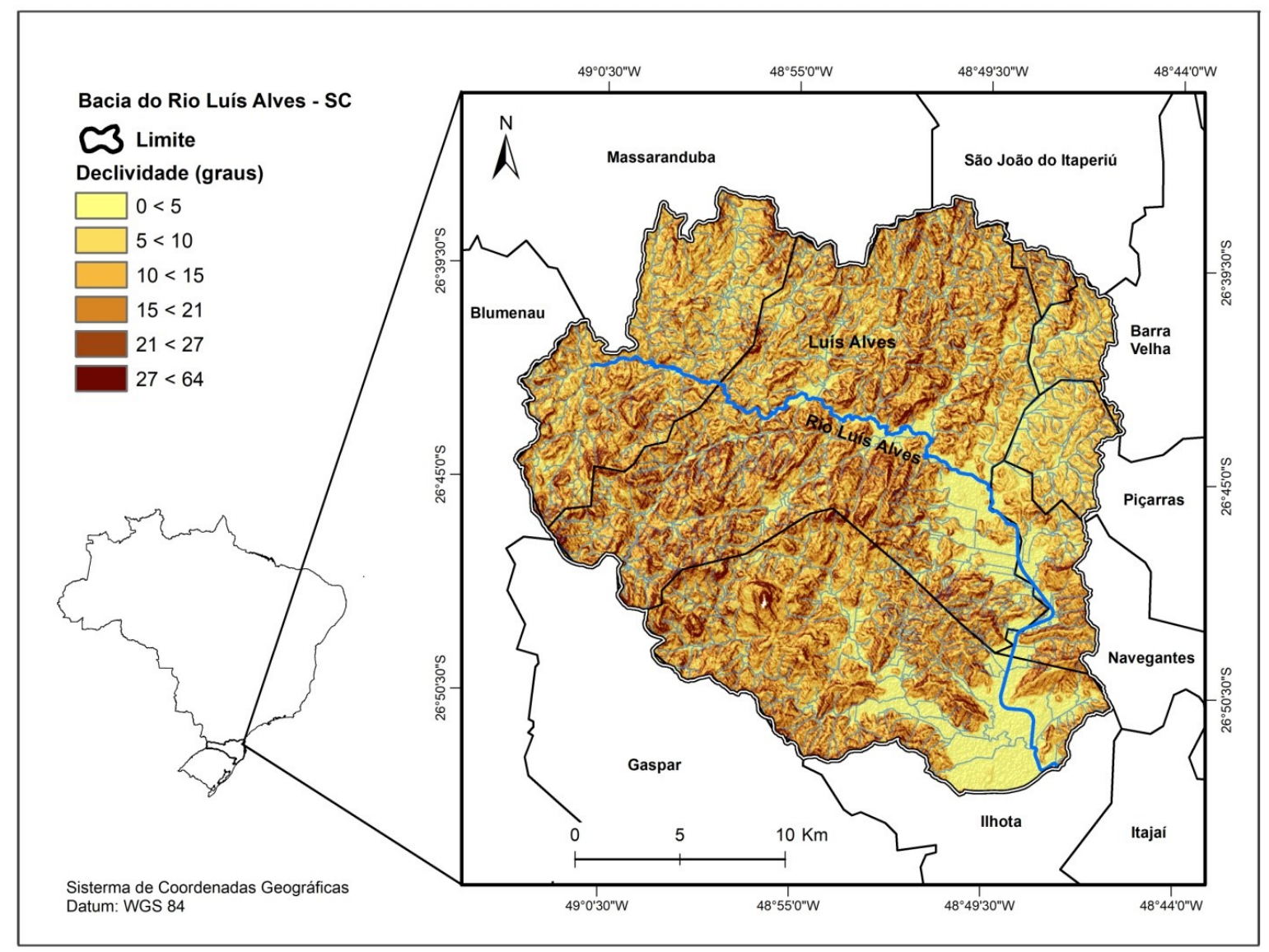

Figura 1: Mapa de localização da área de estudo.

Os eventos associados à ocorrência de movimentos de massa na área de estudo foram devido a um longo período de precipitação, iniciado em meados de agosto de 2008 . O ápice das precipitações foi resultado de uma condição atmosférica, em que a circulação do oceano Atlântico provocou ventos de leste em níveis baixos da atmosfera, a partir de 19 de novembro de 2008. Esta instabilidade atmosférica foi reforçada nos dias 21 a 23 por um vórtice ciclônico, que provou precipitações 
concentradas no Médio Vale do Itajaí. No Vale do Rio Itajaí, que compreende a área de estudo, choveu cerca de $250 \mathrm{~mm} /$ dia entre os dias 23 e 24 de novembro (GERENTE et al., 2015). Os índices pluviométricos registrados na estação de Blumenau, a qual se localiza próxima à área de estudo, foi de $693 \mathrm{~mm}$ acumulados no período de 21 a 25 de novembro (FLORES et al., 2009; SILVA DIAS et al., 2009). Esse evento acarretou em diversos tipos de movimentos de massa na Bacia do Rio Luís Alves, variando entre deslizamentos rotacionais, deslizamentos translacionais e corridas de detritos. Destaca-se também que em razão da grande quantidade de água envolvida, muitos movimentos de massa assumiram um caráter complexo, iniciando-se como deslizamentos e assumindo ao longo de sua zona de transporte um caráter mais fluído, assemelhando-se a corrida de detritos (FLORES et al., 2009; GERENTE et al., 2015).

\section{MATERIAIS E MÉTODOS}

\subsection{Dados}

Neste estudo foram utilizadas 474 cicatrizes de movimentos de massa gerados pelas fortes precipitações no ano de 2008. As cicatrizes foram mapeadas por Luiz et al. (2014) a partir de levantamentos de campo, aliados à interpretação de fotografias aéreas com resolução espacial de 0,39 m obtidas do aerolevantamento fotogramétrico de Santa Catarina disponibilizado pela Secretaria de Estado do Desenvolvimento Econômico Sustentável - SDS (SDS, 2013), realizado entre os anos 2010 e 2011, e em acervos de imagens do Google Earth. A escala do mapeamento das cicatrizes foi compatível a 1:10.000.

Para adequar à metodologia empregada, foram considerados somente os centroides das cicatrizes, ao invés de toda a sua área, denominados neste trabalho de pontos suscetíveis, aos quais se atribuiu valores iguais a 1 (um). Segundo alguns autores, este procedimento evita incertezas relacionadas à delimitação dos limites das cicatrizes e reduz a autocorrelação espacial das amostras (VAN DEN EECKHAUT et al., 2006; PARK e CHI, 2008; PETSCHKO et al., 2012; BRENNING et al., 2015). Além disso, como não está sendo observada a dinâmica do movimento de massa, e sim investigando seus fatores condicionantes, este procedimento fornece tratamento igual para cicatrizes maiores ou menores.

Considerando ainda que a variável resposta é do tipo binária, e para manter a mesma proporção do número de pontos suscetíveis (GOETZ et al., 2011; VORPAHL et al., 2012; PETSCHKO et al., 2014), foram gerados 474 pontos não suscetíveis, com valores iguais a 0 (zero) em locais onde não foram observados movimentos de massa no mesmo período. Isto foi realizado subtraindo da região de estudo as áreas de cicatrizes acrescidas de um buffer de $50 \mathrm{~m}$, medida equivalente a aproximadamente o dobro do tamanho médio das cicatrizes, de forma a evitar que pontos não suscetíveis fossem gerados nos mesmos locais ou muito próximos dos pontos suscetíveis (VORPAHL et al., 2012).

Pourghasemi et al. (2012) ressaltam que a capacidade do modelo de predição da suscetibilidade aos movimentos de massa usualmente é avaliada usando informações independentes, que não foram utilizadas para a construção do modelo. Desta forma, para fins de avaliação do modelo empregado, foram retirados aleatoriamente do conjunto amostral em torno de $5 \%$ de pontos suscetíveis e $5 \%$ de não suscetíveis. Esses pontos serão estimados pelo modelo imposto e depois avaliados (vide Seção 2.3). Assim, a variável resposta ficou composta de 449 pontos suscetíveis e 449 não suscetíveis, com distribuição espacial ilustrada na Figura 2.

Agregado em cada localização da variável resposta há um conjunto de variáveis explicativas, que foram selecionadas como possíveis fatores condicionantes à ocorrência de movimentos de massa. Esta seleção incluiu variáveis Morfométricas, Tipos de solo, Distâncias dos Cursos d'água e das Estradas.

As variáveis Morfométricas consideradas foram: Elevação, Declividade, Orientação de vertentes, Curvatura vertical e Curvatura horizontal. A seleção dessas variáveis foi baseada nas literaturas de Selby (1993), Fernandes e Amaral (2000), Gao e Lo (1995), Lee e Min (2001), Ohlmacher e Davis (2003). Tais autores descrevem a influência dessas variáveis como possíveis fatores condicionantes à ocorrência de eventos de movimentos de massa. Essas variáveis foram obtidas do projeto TOPODATA (VALERIANO, 2008), sendo matrizes (grades) de valores com resolução espacial de 30 $\mathrm{m}$. 


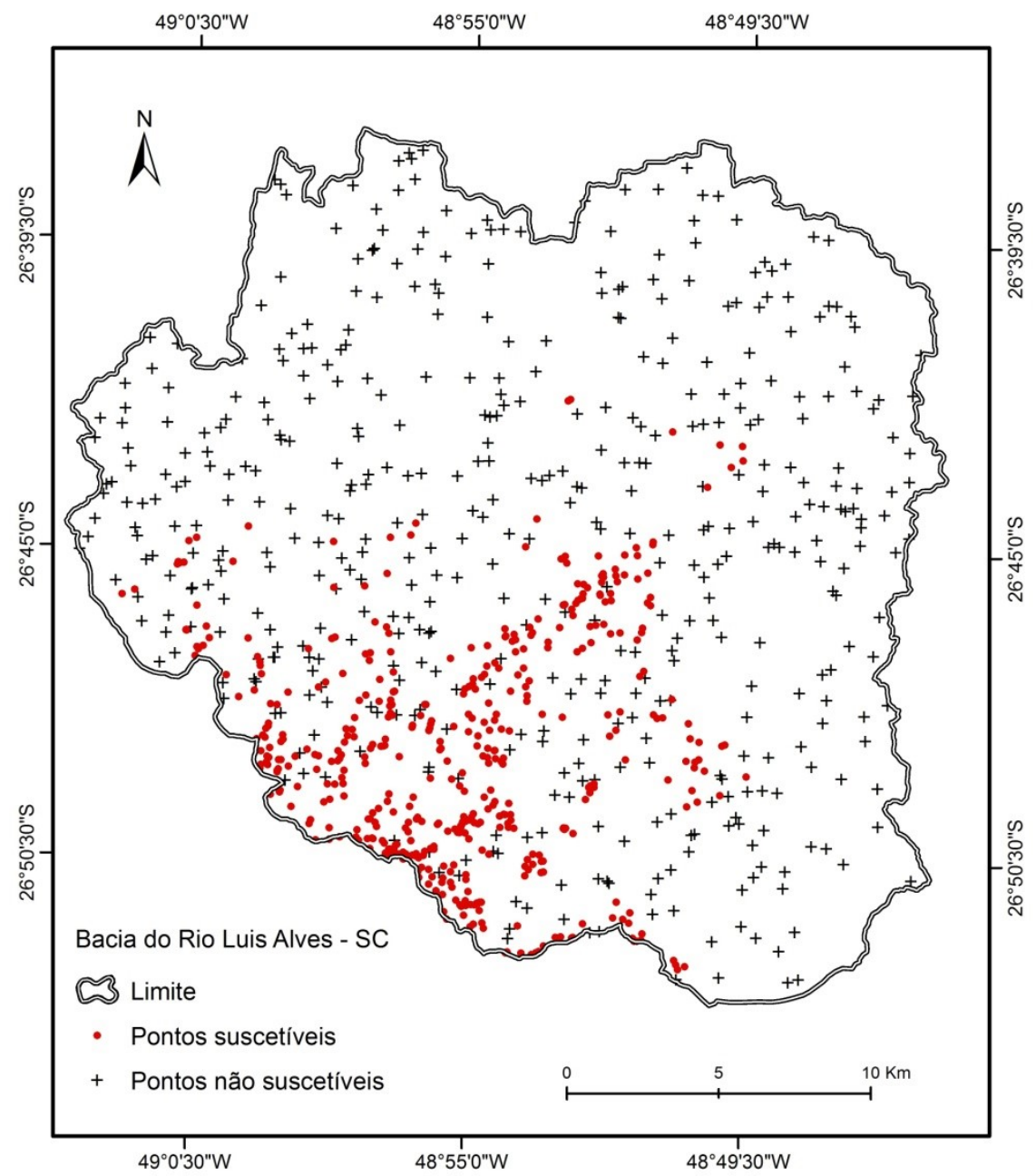

Figura 2: Distribuição espacial da variável resposta na área de estudo.

A inclusão da variável Tipos de solo foi baseada nos trabalhos de Sidle (1985), Crozier (1986) e Selby (1993). A partir do tipo de solo é possível aferir suas características físicas e químicas, tais como ângulo de atrito interno e coesão, as quais possuem influência direta nas tensões do solo. Esta variável foi gerada a partir do mapa de solos (vetorial) da EMBRAPA (2004) na escala de 1:250.000, e posteriormente convertido para matriz de pesos com resolução espacial de $30 \mathrm{~m}$. Os pesos atribuídos para cada tipo de solo, indicados na Tabela 1, foram estabelecidos adotando-se os seguintes critérios: 1) consultas na literatura, como o manual da Embrapa Solos (2004) e Marinho (2014), o qual, ao realizar a estimativa de áreas com potencial de movimentos de massa na Bacia do Rio Luís Alves, apontou que solos do tipo Cambissolo e Litólicos teriam forte influência com a ocorrência de movimentos de massa; 2) cômputo da densidade de cicatrizes de movimento de massa em cada tipo de solo (cicatriz/ $\mathrm{km}^{2}$ ), sendo que se atribuiu maior peso aos solos com maior densidade de cicatrizes e; 3) classe do relevo local de acordo com a Embrapa Solos (2004), sendo atribuído maior peso aos relevos montanhosos e forte ondulado.

Tabela 1 - Atribuição de pesos para a variável Tipos de solo.

\begin{tabular}{lllc}
\hline Tipo de solo & Subtipo & Relevo & Peso \\
\hline Litólico & Ra2 & Montanhoso & 0,9 \\
Cambissolo & Ca32 & Montanhoso & 0,8 \\
Podzólico Vermelho-Amarelo & PVa13 & Forte ondulado & 0,6 \\
Podzólico Vermelho-Amarelo & PVa12 & Ondulado & 0,5 \\
Glei Pouco Húmico Distrófico & HGPd1 & Plano & 0,2 \\
Podzólico Vermelho-Amarelo & PVa8 & Ondulado & 0,1 \\
Glei Pouco Húmico Distrófico & HGPd8 & Plano & 0,1 \\
Podzólico Vermelho-Amarelo & PVa1 & Ondulado & 0,1 \\
\hline
\end{tabular}


A escolha das variáveis Distância dos cursos d'água e Distância das estradas foi baseada nos trabalhos de Temesgen et al. (2001) e Brenning et al. (2015), respectivamente. Essas variáveis foram calculadas a partir da menor distância euclidiana entre cada ponto (suscetível e não suscetível) e os cursos d'água e estradas. A base de dados dos cursos d'água foi obtida a partir da extração automática da rede de drenagem, por meio do programa TerraHidro 0.4.2 (INPE, 2016) utilizando como fonte de dados de entrada a variável Elevação do TOPODATA. A proximidade com os cursos d'água está diretamente vinculada à umidade e à formação de caminhos preferenciais para o escoamento. Em caso de precipitações intensas, os canais fluviais funcionam como verdadeiras vias por onde escoam sedimentos desprendidos das cabeceiras de drenagem. Além disso, erosões fluviais podem aumentar as tensões cisalhantes devido ao aumento do ângulo de declividade de margens de canais. A base de dados das estradas foi obtida através do projeto colaborativo OpenStreetMap. As estradas geralmente são consideradas locais de instabilidade antrópica induzida, pois, para a sua construção, geralmente são realizados cortes no terreno, aumentando assim as tensões de cisalhamento. A Figura 3 sintetiza o procedimento de agregação dos fatores condicionantes à variavel resposta.

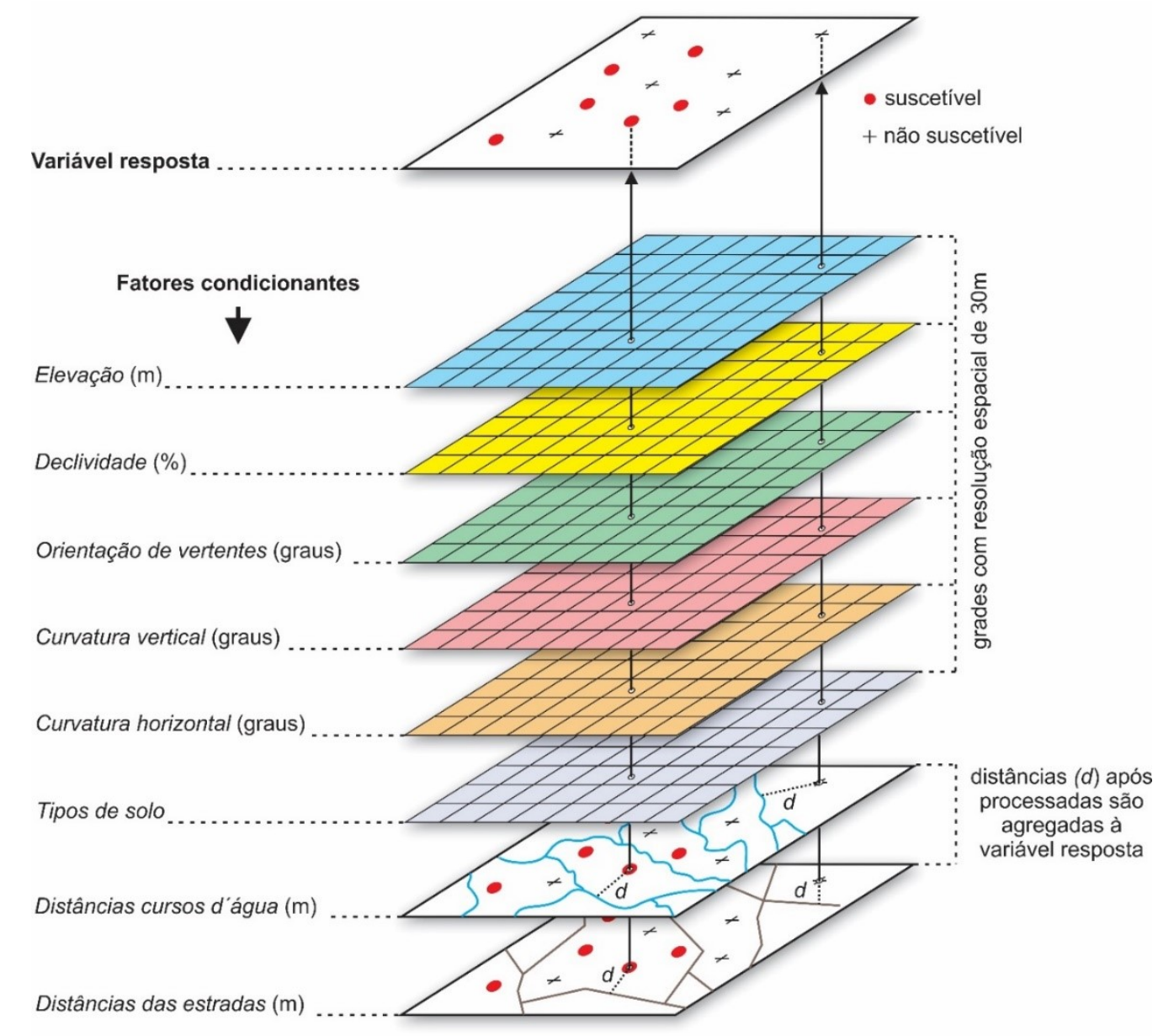

Figura 3: Esquema de agregação dos fatores condicionantes à variavel resposta.

Cabe destacar que, apesar de a precipitação ter sido o principal condicionante da ocorrência de movimentos de massa na região, esta variável não foi incluída no modelo pelo fato de assumi-la constante em toda região de estudo. O evento meteorológico que causou as chuvas extremas nos dias 23 e 24 de novembro de 2008 abrangeu uma grande extensão territorial, sendo registradas inundações e movimentos de massa em diversos munícipios vizinhos a bacia. Além disso, o único registro pluviométrico medido em solo foi o da estação de Blumenau, uma vez que outras estações vizinhas tiveram problemas nos registros em razão da grande magnitude do evento. 


\subsection{Modelo}

A estrutura de modelagem usada é baseada na ideia de processos pontuais espaciais, pelo qual se pode definir e estimar uma medida de suscetibilidade que varia continuamente na região de interesse (KELSALL e DIGGLE, 1998). Este processo define primeiro uma medida de suscetibilidade para detectar as áreas com maiores e menores probabilidades de serem atingidas. Para isto, avaliase simultaneamente a variação na distribuição espacial de pontos suscetíveis quando comparados à distribuição espacial de pontos não suscetíveis.

Considere $\left(s_{1}, s_{2}, \ldots, s_{n_{1}}\right)$ as localizações espaciais de $n_{1}$ pontos suscetíveis e $\left(s_{n_{1}+1}, s_{n_{1}+2}, \ldots, s_{n}\right)$ as localizações espaciais de $\left(n-n_{1}\right)$ pontos não suscetíveis na região de estudo $A \subset \mathrm{R}^{2}$ como sendo observações de dois processos de Poisson I e II, com intensidades $\lambda_{1}(s)$ e $\lambda_{2}(s)$, respectivamente. $\mathrm{O}$ logaritmo da suscetibilidade na localização $s$ é dado por $\rho(s)=\log \left(\lambda_{1}(s) / \lambda_{2}(s)\right)$ e o objetivo na análise é investigar a variação espacial de $\rho(s)$ em $A$ (KELSALL e DIGGLE, 1998).

Seja $y_{i}$ um indicador associado à localização espacial $s_{i}$, tal que: $y_{i}=1$ se $s_{i}$ for um ponto suscetível ou 0 caso contrário. Considera-se que $y_{i}(i=1, \ldots, m)$ são realizações de variáveis aleatórias independentes, $Y_{i} \sim$ Bernoulli $(p(s))$, conforme Kelsall e Diggle (1998) (Equação 1):

$$
p(s)=\frac{q_{1} \lambda_{1}(s)}{q_{1} \lambda_{1}(s)+q_{2} \lambda_{2}(s)} \quad \text { Equação } 1
$$

em que: $q_{1}$ representa a proporção de pontos suscetíveis e $q_{2}$ a de pontos não suscetíveis, em relação ao total existente.

Sob estas considerações Kelsall e Diggle (1998) mostram que (Equação 2):

$$
\operatorname{logit}[p(s)]=\log \left[\frac{p(s)}{1-p(s)}\right]=\rho(s)+c
$$

Equação 2

em que: $c=\log \left(q_{1} / q_{2}\right)$, então $c$ é simplesmente uma constante aditiva, portanto, não modifica as características gerais da distribuição espacial da suscetibilidade sobre a região $A \subset \mathrm{R}^{2}$. Dessa forma, pode-se obter estimativas espaciais de $\rho(s)$, à parte de uma constante aditiva, com um modelo atribuído a variáveis binárias.

Uma extensão natural da abordagem de regressão binária é considerar a inclusão de fatores condicionantes (covariáveis) na regressão. Isso nos leva à abordagem do GAM (HASTIE e TIBSHIRANI, 1990), que pode ser entendido como um modelo linear generalizado que foi estendido por uma componente aditiva espacial que, por suposição, varia suavemente na região de estudo. Um GAM com uma função de ligação logit, conforme sugerido por Kelsall e Diggle (1998) é dado por (Equação 3):

$$
\operatorname{logit}[p(s)]=\log \left[\frac{p(s)}{1-p(s)}\right]=\boldsymbol{\beta} \boldsymbol{x}+g(s)
$$

\section{Equação 3}

em que: $\boldsymbol{x}$ é o vetor de covariáveis, $\boldsymbol{\beta}$ são seus efeitos e a única suposição acerca de $g$ é que esta é uma função suave, porém desconhecida, das coordenadas espaciais $s$. Se a suscetibilidade é considerada constante na região de estudo, então $g(s)=0$ e o modelo reduz-se a um modelo de regressão logística usual (HOSMER e LEMESHOW, 1989).

O procedimento de estimação de $\boldsymbol{\beta}$ e $g(s)$ baseia-se em métodos iterativos usuais de modelos aditivos generalizados, conforme descritos em Hastie e Tibshirani (1990). Neste procedimento, $g(s)$ é estimado usando regressão Kernel ponderada (WAND e JONES, 1995), o qual influencia nas estimativas de $\boldsymbol{\beta}$. 
O modelo proposto (Equação 3) estima uma superfície de suscetibilidade, porém é importante avaliar se esta superfície varia significativamente na região de estudo, ou seja, se existem evidências estatísticas suficientes para rejeitar a hipótese nula de suscetibilidade constante na região $(\mathrm{H} 0: g(s)=0)$. Além disso, é de interesse a construção de contornos de tolerância que auxiliam na identificação de áreas onde a suscetibilidade é significativamente superior ou inferior à média global. O teste global da suscetibilidade e a identificação de áreas de baixa e alta suscetibilidade foram realizados empregando o método de simulação de Monte Carlo proposto por Kelsall e Diggle (1998).

\subsection{Composição e avaliação do modelo}

Antes de avaliar o modelo é necessário definir quais fatores condicionantes podem ou não compor o modelo final. De forma resumida, isto é realizado através de uma análise sequencial, que permite avaliar o efeito do acréscimo de uma ou mais variáveis no modelo. Inicialmente, considera-se todos os fatores condicionantes e o termo $g(s)$; analisa-se o resultado. Seguindo, remove-se um dos fatores condicionantes e novamente analisa-se o resultado. Depois, repõe-se o fator condicionante removido da análise anterior e remove-se outro; analisa-se o resultado. Este processo se repete até que todos os fatores condicionantes tenham sidos avaliados. Finalmente, somente os fatores condicionantes com efeitos $(\beta)$ significantes são considerados para compor o modelo final.

Uma vez estabelecido o modelo final, o mesmo é empregado para estimar uma superfície de suscetibilidade. O método de avaliação empregado neste trabalho consiste em extrair da superfície estimada de suscetibilidade os valores dos pontos suscetíveis e não suscetíveis que foram previamente selecionados do conjunto amostral (vide Seção 3.1). O que se espera é que a distribuição estatística de tais pontos (pontos suscetíveis e não suscetíveis) sejam distintas, com média estimada para os pontos suscetíveis próxima de um (1) e dos não suscetíveis em torno de zero (0). Isto pode ser avaliado graficamente através da construção de um boxplot, que permite uma comparação visual entre dois ou mais grupos.

\section{RESULTADOS E DISCUSSÕES}

$\mathrm{Na}$ primeira análise sequencial, que considerou todas as variáveis explicativas, foi eliminada a variável Curvatura vertical. Nas análises sequenciais posteriores foram eliminadas as variáveis explicativas, na ordem: Curvatura horizontal, Distância das estradas e Orientação de vertentes. Estas variáveis não apresentaram significância estatística para às estimativas de seus respectivos coeficientes $(\beta)$, que pode estar relacionado a alguns aspectos particulares da área estudada, conforme segue:

i) A forma da encosta, expressa pela combinação da Curvatura vertical e horizontal, pode ser considerada como um fator condicionante indireto para deflagração de movimentos de massa, uma vez que influencia na convergencia ou divergencia de fluxo. As variáveis de curvatura utilizadas neste trabalho foram inseridas no modelo em sua forma bruta, com resolução original de $30 \mathrm{~m}$, ou seja, sem nenhum tratamento para sua agregação. Desta forma, elas apresentaram uma grande variabilidade espacial, gerando um aspecto ruidoso aos dados, dificultando assim a extração de informações contínuas ao longo da área de estudo, bem como a correlação entre as ocorrências;

ii) os movimentos de massa que ocorrem próximo a estradas geralmente são deslizamentos rotacionais, os quais geralmente ocorrem em locais com manto de alteração mais profundo e em geral possuem extensões menores do que outros tipos, tais como corridas de detrito. Apesar do uso de ortofotos de altíssima resolução espacial $(0,39 \mathrm{~m})$ para o mapeamento das cicatrizes, no momento do mapeamento manteve-se uma escala de 1:10.000, o que pode ter prejudicado a detecção de cicatrizes menores. Além disso, devido à defasagem temporal de dois anos entre a aquisição das ortofotos usadas para o mapeamento das cicatrizes, e o evento que desencadeou os movimentos de massa, é possível que a vegetação das cicatrizes menores tenha se regenerado, dificultando seu mapeamento. Isso corrobora com o fato desta variável ter sido descartada pelas análises estatísticas;

iii) a Orientação de vertentes é uma variável que possui efeito indireto em termos de condicionar a ocorrência de movimentos de massa. Essa variável tem relação com a intensidade de radiação solar incidente e com o efeito orográfico da chuva, o que pode ser um dos motivos de ela não ter sido significativa no modelo gerado, uma vez que a chuva foi constante e bem distribuída em toda a bacia. 
A Tabela 2 sintetiza o resultado da análise sequencial efetuada com seus os respectivos fatores condicionantes.

Tabela 2: Estimativas dos coeficientes de regressão com suas respectivas significâncias.

\begin{tabular}{lcccc}
\hline \multicolumn{1}{c}{$\boldsymbol{\beta}$} & Estimativa & Erro padrão & $\boldsymbol{t}$-valor & $\boldsymbol{p}$-valor \\
\hline Intercepto $\left(\beta_{0}\right)$ & $-5,8275$ & 1,0785 & $-5,4029$ & $8,3761 \mathrm{e}-08$ \\
Elevação & 0,0041 & 0,0018 & 2,2832 & $2,2603 \mathrm{e}-02$ \\
Declividade & 0,0488 & 0,0183 & 2,6592 & $0,7941 \mathrm{e}-02$ \\
Tipos de Solo & 5,0538 & 1,1852 & 4,2639 & $2,2180 \mathrm{e}-05$ \\
Distância Cursos d'água & $-0,0027$ & 0,0012 & $-2,2069$ & $2,7501 \mathrm{e}-02$ \\
\hline
\end{tabular}

Decorrente dos resultados apresentados na Tabela 2, o modelo espacial final aplicado neste estudo é dado por (Equação 4):

$$
\begin{aligned}
\operatorname{logit}[p(s)]=\beta_{0} & +\beta_{\text {Elevação }}+\beta_{\text {Declividade }}+\beta_{\text {Tipos Solo }}+ \\
& +\beta_{\text {Dist. cursos d'agua }}+g(s)
\end{aligned}
$$

Em seguida, o modelo da Equação 4 foi empregado para mapear a distribuição espacial da suscetibilidade a movimentos de massa na área de estudo. O resultado é uma superficie contínua de suscetibilidade, que varia suavemente de um local a outro. Depois, foram realizadas 500 simulações, via método de Monte Carlo, conforme proposto por Kelsall e Diggle (1998), cujos resultados observados foram: i) houve uma variação espacial global significativa da suscetibilidade associada a movimentos de massa ( $p$-valor $=0,001992$ ), portanto a hipótese nula de suscetibilidade constante na região $(g(s)=0)$ foi descartada; ii) a identificação de áreas delimitadas por linhas de contorno de $2,5 \%$ e $97,5 \%$, de aproximadamente $95 \%$ de tolerância, apontam onde a suscetibilidade é significativamente inferior ou superior à média global, respectivamente. O resultado final é o mapa ilustrado na Figura 4.

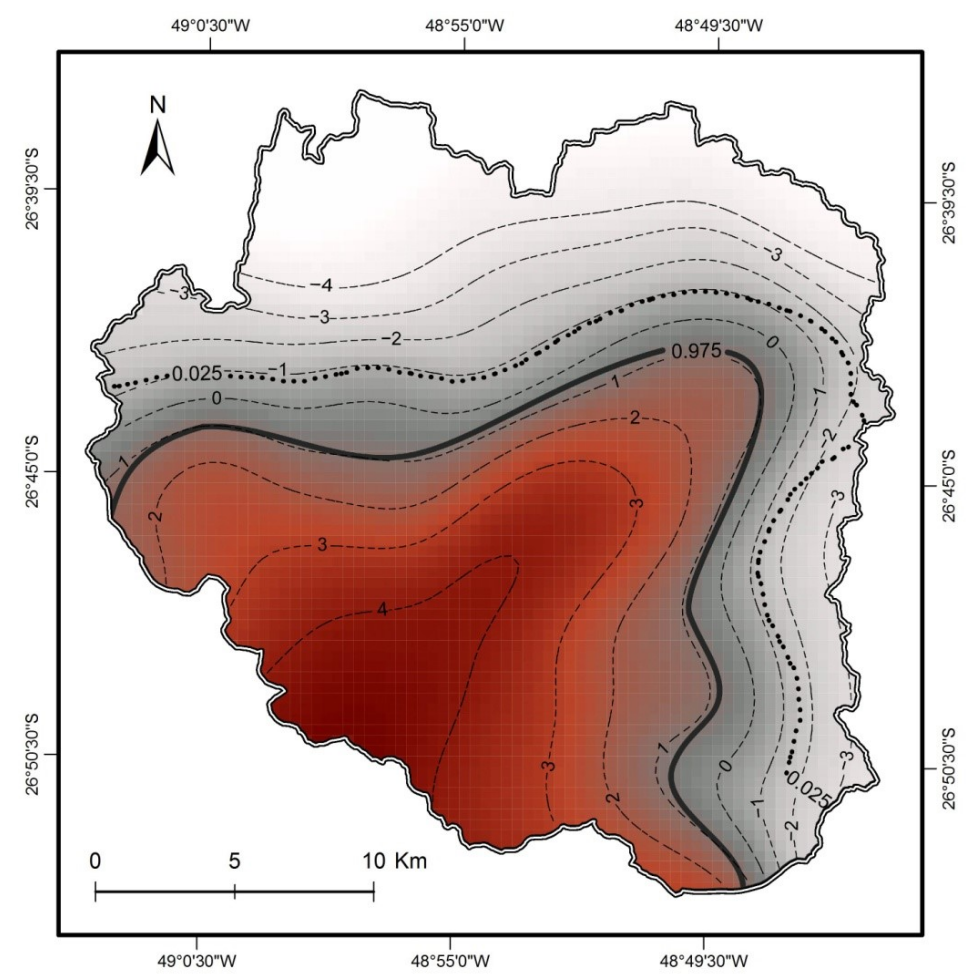

Bacia do Rio Luís Alves - SC

( 3 Limite

Suscetibilidade

logit Prob.

$4 \longdiv { 0 . 9 8 2 0 }$

30.9526

20.8808

$1 \quad 0.7311$

00.5

\begin{tabular}{l|l}
-1 & 0.2689
\end{tabular}

\begin{tabular}{l|l}
-2 & 0.1192
\end{tabular}

\begin{tabular}{l|l}
-3 & 0.0474
\end{tabular}

$-4 \quad 0.0180$

Figura 4: Mapa da suscetibilidade a movimentos de massa na Bacia do Rio Luís Alves. 
Com base no mapa da Figura 4 verifica-se que a região de maior suscetibilidade à ocorrência de movimentos de massa corresponde ao local que possuía a maior densidade de cicatrizes no evento ocorrido em 2008. Essa área está localizada a sudoeste da bacia hidrográfica, representada no mapa em tons avermelhados, a qual possui maiores valores de elevação e é mais declivosa do que o restante da bacia (Figura 1).

Segundo a literatura, quanto mais elevado o valor da elevação, associado a um gradiente altitudinal, maior será a energia cinética aplicada às vertentes e, consequentemente, maior é a capacidade de deslocamento de material, principalmente se associada a elevadas declividades e dissecação (CHRISTOFOLETTI, 1974; THOMAS, 1979). Já a declividade influencia no aumento da tensão de cisalhamento dos materiais da encosta, na velocidade do deslocamento e capacidade de transporte do material mobilizado em uma ruptura ou deformação (SELBY, 1993).

Na área mais suscetível da bacia predominam os tipos de solo Ra2 e Ca32, solos que geralmente são localizados em relevos montanhosos, escarpados e forte ondulados. O solo Ra2 é uma associação entre os solos Litólicos Álicos, textura argilosa, com Cambissolo Álico, possui pequena profundidade e alta pedregosidade (EMBRAPA, 2004). Já o solo Ca32 é uma associação de Cambissolo Álico (textura argilosa e relevo montanhoso) com solos Litólicos Distróficos, de textura argilosa, relevo montanhoso e escarpado. Verifica-se que ambos os tipos de solo são associações entre solos Litólicos com Cambissolo os quais podem ter forte relação com a suscetibilidade do terreno à ocorrência de movimentos de massa do tipo deslizamento (MARINHO, 2014).

A distância dos cursos d'água também foi significativa com a ocorrência de movimentos de massa. A proximidade da encosta às estruturas de drenagem pode ser considerada um fator importante em termos de instabilidade. Durante fortes precipirações, o fluxo de água desses cursos aumentam podendo favorecer a erosão basal e lateral dos sulcos presentes nas encostas (GOKCEOGLU e AKSOY, 1996). Temesgen et al. (2001) afirmam que a proximidade com os cursos d'água está diretamente vinculada à forma da encosta, à umidade e à formação de sulcos e ravinas, ou seja, caminhos preferenciais de escoamento superficial. Nestas áreas, as encostas apresentam-se geralmente em seções côncavas, que, por serem zonas de convergência de fluxos d'água (superficiais e subsuperficiais), favorecem a condição de saturação do solo e intensificam a atuação dos processos erosivos, podendo favorecer a ocorrência de movimentos de massa.

Verifica-se que algumas dessas variáveis explicativas também foram mencionadas por outros autores na análise da suscetibilidade a movimentos de massa. Ayalew e Yamagishi (2005) encontraram que, entre os parâmetros morfométricos, a orientação (ou aspecto) teve um bom papel no modelo logístico gerado, seguido da declividade. A elevação, segundo eles, não foi um bom estimador para o modelo. No entanto, os autores atrelaram isso ao fato de que o papel dessa variável já havia sido enfatizado em outras duas variáveis (cortes de estradas e relação rocha-leito-inclinação) correlacionadas com a elevação. Cabe ainda salientar que Ayalew e Yamagishi (2005) utilizaram a regressão logística que, a elevação nem sempre manterá uma relação log-linear com a ocorrência de movimentos de massa, o que também foi apontado por Park e Chi (2008).

Goetz et al. (2011) ao verificar a suscetibilidade de movimentos de uma bacia hidrográfica em Vancouver, Canadá, usando o GAM, GLM e modelos físicos, associados a variáveis do terreno e dados de uso da terra, encontraram que a curvatura do plano e do perfil, além da declividade, foram consideradas importantes modificadores da estabilidade das encostas. Brenning et al. (2015), utilizando o GAM, e Ayalew e Yamagishi (2005), utilizando o GLM, encontraram forte correlação entre a ocorrência de movimentos de massa e a proximidade com as estradas, o que não foi observado no presente estudo. Contudo, as estradas existentes na área de estudo deste trabalho não são de grande magnitude, enquanto que nos estudos dos autores supramencionados tratava-se de grandes rodovias, que podem acarretar maior impacto no terreno, sobretudo em razão das vibrações geradas pelos automóveis. 
A partir do mapa de suscetibilidade foram extraídos os valores de suscetibilidade estimada para o conjunto de amostras selecionadas para ambas as classes avaliadas (pontos suscetíveis e não suscetíveis reservados para avaliação). O resultado desta avaliação é apresentado na Figura 5.

Conforme a Figura 5 observa-se que a suscetibilidade estimada é bastante distinta. Pode-se afirmar que $100 \%$ das amostras relativas às áreas suscetíveis apresentam suscetibilidade estimada superiores a 0,9. Por outro lado, áreas não suscetíveis apresentaram uma maior variação de valores de suscetibilidade estimado, sendo que cerca de $75 \%$ das amostras desta classe apresentaram valores inferiores a 0,5 . Isto pode ser uma indicação de que o modelo empregado possui boa capacidade de predição da suscetibilidade à ocorrência de massa da área de estudo.

Outros autores também encontraram bons resultados ao se utilizar o GAM para a modelagem de suscetibilidade a movimentos de massa. Brenning et al. (2008) utilizaram o GAM para gerar mapas de suscetibilidade a movimentos de massa nos Andes Equatorianos, relacionando uma série de variáveis morfométricas e de uso da terra. A avaliação dos modelos dos autores indicou valores de AUROC (Area Under a Receiver Operating Characteristic Curve) entre 0,67 e 0,77. Goetz et al. (2011), ao compararem modelos físicos e empíricos para modelar a suscetibilidade a movimentos de massa na região de Vancouver, Canadá, relataram que o GAM apresentou melhores resultados (AUROC de até 0,808 ) que os demais métodos testados, como o GLM e SHALSTAB. Brenning et al. (2015) utilizaram o GAM e o GLM para avaliar a suscetibilidade a deslizamentos próximo a grandes rodovias nos Andes, sul do Equador, encontrando AUROC de até 0,81 com o GAM.

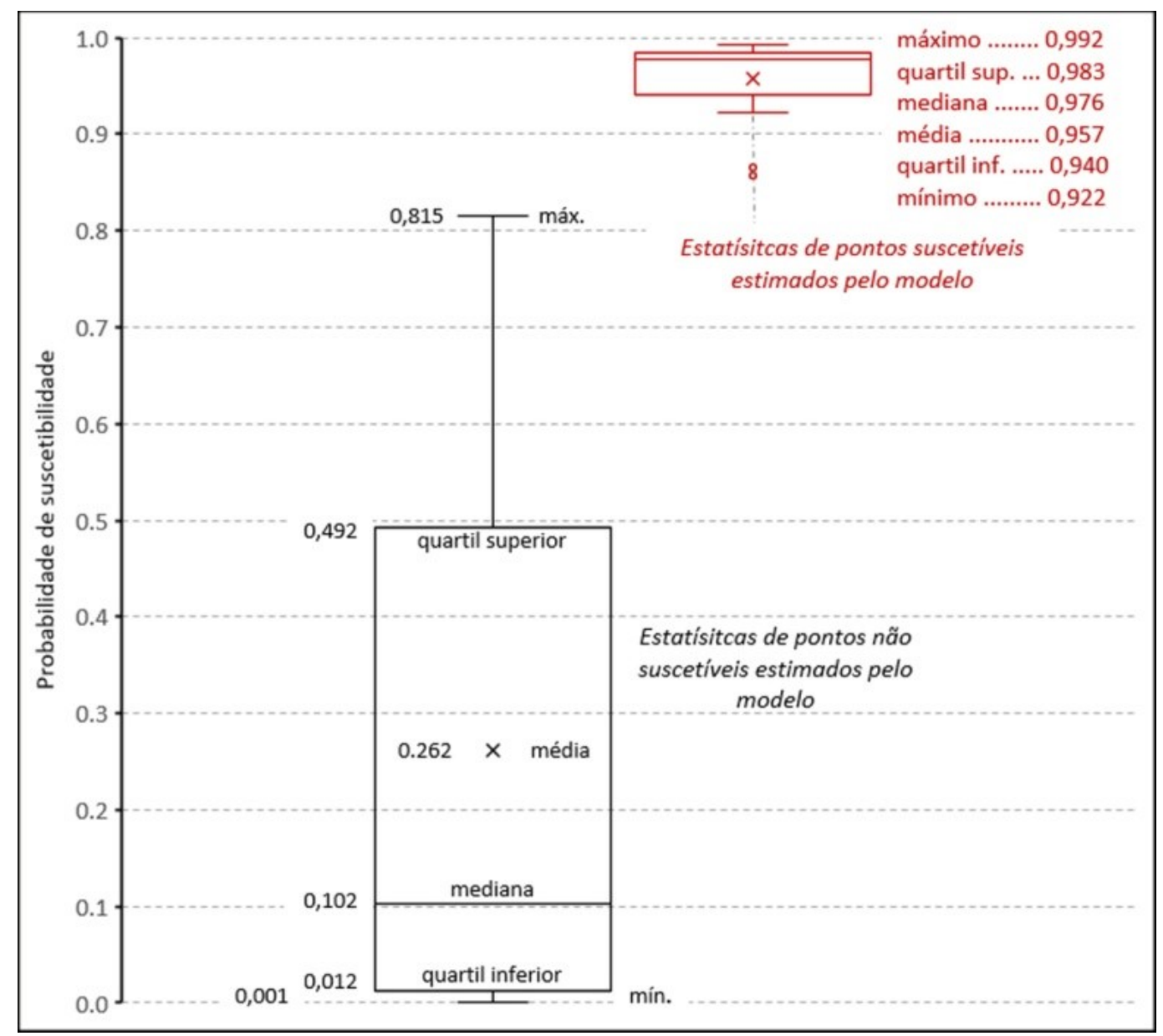

Figura 5: Avaliação da suscetibilidade estimada pelo modelo espacial. 


\section{CONSIDERAÇÕES FINAIS}

Este trabalho mostrou ser possível, com base na qualidade e disponibilidade de informações pontuais, obter estimativas da suscetibilidade a movimentos de massa continuamente no espaço, controlado por fatores condicionantes. Neste caso, as variáveis Declividade, Elevação, Tipos de solo e Distância dos cursos d'água foram considerados fatores condicionantes significativos no modelo de suscetibilidade a movimentos de massa na área de estudo.

A avaliação do mapa de suscetibilidade gerada pelo modelo mostrou que $100 \%$ dos pontos considerados suscetíveis apresentaram suscetibilidade estimada superiores a 0,9, indicando que 0 modelo fez uma boa predição da suscetibilidade do local. Ao identificar as áreas em que a suscetibilidade à ocorrência de movimentos de massa é significativamente maior, o modelo empregado possibilita o planejamento de ações mais específicas e dirigidas a essas áreas.

Um desdobramento importante seria a comparação da superfície de suscetibilidade obtida neste estudo com outros estudos, nos quais se pudessem detectar outras particularidades das áreas em suscetibilidade. Isto permitiria um avanço nas análises, fornecendo mais elementos para compreensão do evento investigado e, assim reduzir as incertezas associadas à predição e ampliar a capacidade de resposta das instituições responsáveis pelo monitoramento e alerta para situações de suscetibilidade a movimentos de massa. Para trabalhos futuros recomenda-se testar a influência da variação dos dados de entrada como, por exemplo, diferentes escalas e outras variáveis nas respostas do modelo.

\section{REFERÊNCIAS}

ALVES, M.P.A. Análise comparativa dos fatores ambientais nos eventos de inundações na bacia hidrográfica do vale do Itajaí: Novembro de 2008 e Setembro de 2011. Revista Discente Expressões Geográficas, v. 09, p. 173, 2014.

AYALEW, L., YAMAGISHI, H. The application of GIS-based logistic regression for landslide susceptibility mapping in the Kakuda-Yahiko Mountains, Central Japan. Geomorphology, v. 65, p. 12-31, 2005.

BASTOS, F.H.; PEULVAST, J.P. Suscetibilidade à Ocorrência de Movimentos de Massa no Maciço de Baturité - Ceará, Brasil. Revista do Departamento de Geografia USP, v. 32, p. 124-142, 2016.

BRENNING, A.; SCHWINN, M.; RUIZ-PÁEZ, A.P.; MUENCHOW, J. Landslide susceptibility near highways is increased by 1 order of magnitude in the Andes of southern Ecuador, Loja province. Natural Hazards and Earth System Sciences, v. 15, p. 45-57, 2015.

BRENNING, A.: Improved spatial analysis and prediction of landslide susceptibility: Practical recommendations, in Landslides and Engineered Slopes, Protecting Society through Improved Understanding, edited by: Eberhardt, E., Froese, C., Turner, A. K., and Leroueil, S., Taylor \& Francis, Banff, Alberta, Canada., p. 789-795, 2012.

BRENNING, A. Statistical geocomputing combining R and SAGA: the example of landslide susceptibility analysis with generalized additive models. In: Böhner, J., Blaschke, T., Montanarella, L. (Eds.), SAGA

- Seconds Out Hamburger Beiträge zur Physischen Geographie und Landschaftsökologie, v. 19, p. 23-32, 2008.

BRENNING, A. Spatial prediction models for landslide hazards: review, comparison and evaluation, Natural Hazards and Earth System Sciences, v. 5, p. 853-862, 2005.

CARDOSO, C. S. de; CRUZ, G. S. de ; CALEARO, D. S. Análise da Tromba d'Água de 02 de março de 2008 em Florianópolis/SC. 2008. In: XV CONGRESSO BRASILEIRO DE METEOROLOGIA, 2008. São Paulo, 2008.

CHRISTOFOLETTI, A. Geomorfologia. São Paulo: Edgard Blucher, 1974. 149 p.

CROZIER, M.J. Landslides: causes, consequences and environment. Croom Helm, London, 1986.

EMBRAPA. Solos de Santa Catarina. Boletim de Pesquisa e Desenvolvimento 46. ISSN 1678-0892, 2004. 
FERNANDES, N.F.; AMARAL, C.P. Movimentos de Massa: Uma abordagem geológico-geomorfológica. In: Guerra, A. J. T e Cunhas, S. B. (orgs). Geomorfologia e Meio Ambiente. 3 ed., Bertrand Brasil, 2000. 372p.

FLORES, J. A. A.; PELLERIN, J. R. G. M.; ÉGAS, H. M. Movimentos Gravitacionais de Massa no Município de Gaspar, Vale do Itajaí, SC, na catástrofe de novembro de 2008. Caracterização dos processos por critérios geomorfológicos, geológicos e pedológicos. In: XIII Simpósio Brasileiro de Geografia Física Aplicada, 2009, Viçosa. XIII Simpósio Brasileiro de Geografia Física Aplicada, 2009.

GALL, M.; NGUYEN, K.; CUTTER, S.L. Integrated research on disaster risk: is it really integrated? International Journal of Disaster Risk Reduction, v. 12, p. 255-267, 2015.

GAO, J.; LO, C.P. Micro-scale modelling of terrain susceptibility to landsliding from a DEM: a GIS approach. Geocarto International, v. 10, n. 4, p. 15-30, 1995.

GERENTE, J.; BINI, G. M. P., LUIZ, E. L. Análise da variável forma de relevo como condicionante de movimentos de massa: estudo de caso na Bacia do Rio Saltinho em Gaspar/SC. Revista Equador (UFPI), v. 4, n. 3, 2015.

GOKCEOGLU, C.; AKSOY, H. Landslide susceptibility mapping of the slopes in the residual soils of the Mengen region (Turkey) by deterministic stability analyses and image processing techniques. Eng Geol, v. 44, p. 147-161, 1996.

GOETZ, J.N.; GUTHRIE, R.H.; BRENNING, A. Integrating physical and empirical landslide susceptibility models using generalized additive models, Geomorphology, v. 129, p. 376-386, 2011.

GUZZETTI, F.; CARRARRA, A.; CARDINALI, M.; REICHENBACH, P. Landslide hazard evaluation: a review of current techniques and their application in a multi-scale study, central Italy. Geomorphology, v. 31, p. 181-216, 1999.

HASTIE, T.J.; TIBSHIRANI, R.J. Generalized Additive Models. 1 ed. London; New York: Chapman and Hall, 1990. 335p.

INSTITUTO NACIONAL DE PESQUISAS ESPACIAIS, INPE. 2016. TerraHidro. Disponível em: <http://wiki.dpi.inpe.br/doku.php?id=terrahidro> Acesso em: 20 de nov. de 2016.

KELSALL J.E.; DIGGLE P.J. Spatial variation in risk of disease: a nonparametric binary regression approach. Journal of the Royal Statistical Society Series C Applied Statistics, v. 47, P. 559-573, 1998.

LEE, S.; MIN, K. Statistical analysis of landslide susceptibility at Yongin, Korea. Environmental Geology, v. 40, p. 1095-1113, 2001.

LUIZ, E.L.; GASPER, B.; GERENTE, J. Movimentos de massa na bacia do rio Luís Alves e sua relação com zonas de saturação e declividade dos terrenos. 2014. Relatório de pesquisa.

MARINHO, R. Estimativa de áreas com potencial de deslizamento de terra na região do Morro do Baú, Santa Catarina, através do modelo de relação de frequência e SIG. Revista Geonorte, Edição Especial 4, v.10, n.1, p.136-140, 2014.

OHLMACHER C. G; DAVIS C. J. Using multiple regression and GIS technology to predict landslide hazard in northeast Kansas, USA. Engineering Geology, v. 69, p. 331-343, 2003.

PARK, N.-W.; CHI, K.-H. Quantitative assessment of landslide susceptibility using high-resolution remote sensing data and a generalized additive model, Int. J. Remote Sens., v. 29, p. 247-264, 2008.

PETSCHKO, H.; BELL, R., BRENNING, A.; GLADE, T. In: EBERHARDT E.; FROESE C.; TURNER A.K.; LEROUEIL S. (Eds.). Landslide susceptibility modeling with generalized additive models - facing the heterogeneity of large regions Landslides and Engineered Slopes, Taylor \& Francis, p. 769-777, 2012.

PETSCHKO, H.; BRENNING, A.; BELL, R.; GOETZ, J.; GLADE, T. Assessing the quality of landslide susceptibility maps - case study Lower Austria. Natural Hazards and Earth System Sciences, v. 14, n. 95-118, 2014. 
PINTO, R. C.; PASSOS, E.; CANEPARO, S. C. Classificação dos movimentos de massa ocorridos em março de 2011 na Serra da Prata, estado do Paraná. Geoingá: Revista do Programa de PósGraduação em Geografia, v. 4, n. 1, p. 3-27, 2012.

POURGHASEMI, H. R.; PRADHAN, B.; GOKCEOGLU C.; MOEZZI, D. Landslide Susceptibility Mapping Using a Spatial Multi Criteria Evaluation Model at Haraz Watershed, Iran. In:

Terrigenous Mass Movements. B. Pradhan and M. Buchroithner (eds.), Springer-Verlag Berlin Heidelberg, 2012.

R Development Core Team (2009). R: A language and environment for statistical computing. R Foundation for Statistical Computing, Vienna, Austria. ISBN 3-900051-07-0, http://www.R-project.org.

SECRETARIA DE ESTADO DO DESENVOLVIMENTO ECONÔMICO SUSTENTÁVEL - SDS. Sistema de Informações Geográficas de Santa Catarina (SIGSC). Disponível em: <http://sigsc.sds.sc.gov.br/>. Acesso em: 12 de nov. de 2013.

SELBY, M. J. Hillslope Materials and Process. New York: Oxford University Press, 1993. 451p

SIDLE, R. C.; PEARCE, A. J.; LOUGHLIN, C. L. O. Hillslope stability and landuse. American geophysical union, Washington DC, USA, p. 125, 1985.

SILVA DIAS, M. A. F. (Ed.). As chuvas de novembro de 2008 em Santa Catarina: um estudo de caso visando à melhoria do monitoramento e da previsão de eventos extremos. Nota técnica. São José dos Campos, INPE. 2009, 67p.

TEMESGEN, B.; MOHAMMED, M. U.; KORME, T. Natural hazard assessment using GIS and remote sensing methods, with particular reference to the landslides in the Wondogenet Area, Ethiopia. Physics and Chemistry of the Earth, v. 26, p. 615-665, 2001.

THOMAS, M. F. Tropical geomorphology: a study of weathering and landform development in warm climates. 2 ed. London: MacMillan, 1979. 332p

VALERIANO, M. M. TOPODATA: guia para utilização de dados geomorfológicos locais. INPE, São José dos Campos, 2008. Disponível em: <http://urlib.net/8JMKD3MGP8W/33EPEBL>. Acesso em: 12 de jan. de 2017.

VAN DEN EECKHAUT, M., VANWALlegheM, T., POESEN, J., GOVERS, G., VERSTRAETEN, G., AND VANDEKERCKHOVE, L. Prediction of landslide susceptibility using rare events logistic regression: a case-study in the Flemish Ardennes (Belgium), Geomorphology, v. 76, p. 392-410, 2006.

VORPAHL, P., ELSENBEER, H., MÄRKER, M., AND SCHRÖDER, B. How can statistical models help to determine driving factors of landslides?. Ecol. Model., v. 239, p. 27-39, 2012.

WAND, M. P.; JONES, M. C. Kernel Smoothing. London: Chapman and Hall/CRC. 1994. 224p. 